\title{
Der philosophische Zweifel
}

\author{
Kleine Notiz zu einer grossen Erzählung
}

Georg Kohler

Zweifel sind Primärwerkzeuge der Moderne - und ihr Kainszeichen. Cartesius' Rekonstruktion der Welt aus dem Geist der unbezweifelbaren Gewissheit nutzt den Zweifel zur Wegbereitung des Fortschritts, und noch die »Logik der Forschung « Poppers verwendet das Instrument der systematisch zweifelnden Anti-Verifikation, um dem Erreichten den Glanz wissenschaftlich stabiler Erkenntnis zu sichern - oder genauer: (mehr oder weniger unverhohlen) die Würde, uns auf den Pfad zur "Wahrheit« zu lenken. Die Gegenaufklärer, die auch ein Produkt der Moderne sind, sehen es umgekehrt: Es sind der Zweifel, die durch ihn bewirkte Unsicherheit und Anomie, die Zerrüttung fester Werte und alter Glaubensmächte, es ist die rücksichtslos gewordene Freiheit, an nichts mehr glauben zu müssen, und es ist die neuzeitliche Selbstvergottung der menschlichen Vernunft, die "Gott töten« und den Menschen nicht zum Übermenschen, aber zum Un-Tier und schwarmsüchtigen Massenwesen gemacht haben. So lautet das Verdikt aller Reaktionäre - von Joseph de Maistre, Dostojewsky bis zu den religiösen Fundamentalisten unserer Tage und jeglicher Provenienz. - Eine Gesellschaft, von der ich mich fernhalten möchte.

Anderseits: Wer nachdenkt über Zweifel und Zweifeln, muss aufpassen, nicht zum naiven Ritter der rationalistischen Moderne und ihrer Ansprüche zu werden. Frau Moderne liebt es nämlich, ihre Abstammung und deren Wurzeln zu verschweigen; sind sie doch nicht nur mit "kritischer Vernunft", sondern ebenso mit Glaube, (Selbst)Liebe und Hoffahrt verschwistert. Unbewiesene und unbeweisbare Voraussetzungen, "Glauben" und Zweifelsverweigerung gehören zum elementaren Selbstbewusstsein der Moderne, und man darf sich fragen, wie zeitgemäss dieses Bewusstsein selber noch ist.

Die Frage ist freilich allzu gross, um im Rahmen meiner kleinen Zweifelsnotiz mehr als eine Frage zu bleiben. Ein Stück weit ist sie gleichwohl zu vertiefen. Einiges zum Zweifeln und zum Zweifel lässt sich, umrissweise, dadurch vielleicht doch erfahren. 
Drei Überlegungsfelder bieten sich an: Das eine liefert Merkmale der Sache durch die Reflexion auf die Stufenreihe vom alltäglichen Fragen bis zur totalen Skepsis. Das zweite umgrenzt die existentielle Bedeutung des Zweifelns für das Identitätsgefühl, d.h. für das Selbstbewusstsein der zurechnungsfähigen Person. Das dritte eröffnet sich, wenn man überlegt, wie viel transrationale Entzweifelungen immer schon nötig sind, um - zum Beispiel - zur ästhetischen Wahrnehmung fähig zu sein.

Auf das zuletzt Genannte möchte ich zunächst hinweisen, um dann den ersten Aspekt zu behandeln. Der an zweiter Stelle erwähnte Bereich sei im Rahmen dieser knappen Skizze der Psychologie und der Psychopathologie überlassen.

Ästhetisches Wahrnehmen, das Vermögen, sich von etwas in einer Weise berühren zu lassen, die "viel zu denken" ermöglicht, aber nichts ein für allemal "auf den Begriff zu bringen" erlaubt (um an kantische Beschreibungen - in der "Kritik der ästhetischen Urteilskraft" zu erinnern), braucht, als Bedingung seiner Möglichkeit, die Grenzüberschreitung allzu rigider Rationalitätsgebote. Wer nicht zu phantasieren, wer nicht zu träumen imstande ist, wer sich nicht von der "apparition", der "Erscheinung" des Inkommensurabeln im Kunstwerk oder im Dasein des Naturschönen, verwandeln lassen kann, der wird - gerade als dezidiert »Moderner» - ästhetisch taub bleiben.

Bei der Vorbereitung dieses Textes bin ich auf eine Anekdote gestossen, die ich hier gerne wiedergebe. Sie betrifft den berühmten Philologen und Gräzisten Wilamowitz-Möllendorf, der unbestreitbar ein moderner Wissenschafter (wenn auch einer des "Geistes«) war und der ohne weiteres als zweifelsfähiger Zeitgenosse gelten darf:

»Er könne, bemerkt (Wilamowitz, in: Der Glaube der Hellenen), nicht mit Zuversicht die Möglichkeit bestreiten, dass Herodots Bericht über das angebliche Auftreten der Göttin Athene in Begleitung des siegreichen Peisistratos wahr sei. [...] Ja, es fällt Wilamowitz keineswegs schwer, sich in solche Begegnungen hineinzuversetzen. >Ich selbst , berichtet er, `habe eine Epiphanie von ihm - er meint den Flurgott Pan - serlebt, als ich in einem Hohlweg Arkadiens ritt und plötzlich über meinem Kopf in den 
Ästen eines Baumes ein merkwürdiger Bock erschien und ohne sich zu rühren auf Ross und Reiter unter sich herabsah.«

Kurt Hübner, dem ich den Hinweis verdanke, diskutiert Wilamowitz' Bekenntnis im Kontext seiner Darstellung des Mythos, wonach Menschen nie - daher auch nicht in der Epoche rationaler Entzauberung der Wirklichkeit - darauf verzichten können, die lebensweltliche Realität, die Begegnungen mit der Natur und den Mitmenschen, mit Liebe, Geburt, Tod und anderen Schlüsselgeschehnissen humaner Existenz, auf eine mythische/ mythologische, mit Narrativen und personalen Kräften operierende Weise zu erfahren. Als Verteidiger dieser Vermutung zitiert er u.a. den einflussreichen französischen Kulturhistoriker Jean-Pierre Vernant, der von der prinzipiellen Kluft ausgeht, die zwischen "Mythos und Logos" aufklaffe:

"Der Dialog sei unmöglich [...] Selbst wenn [Mythos und Logos] dasselbe Objekt zu betrachten, in die gleiche Richtung zu zeigen scheinen, es bleiben die beiden Arten der Rede für einander undurchdringlich. Wählt man den einen Sprachtyp, so nimmt man zugleich vom anderen Abschied. $\ll^{2}$

So verstanden hat das mythisch begründete Erleben seine eigene Struktur und Semantik, seine eigene - transrationale - Logik.

Ich will das Thema "Mythos, Logos, Rationalität der Moderne« nicht länger verfolgen. Wesentlich ist bloss die zweifache Aussage, dass es immer wieder notwendig sein wird, eine von der Rationalität in ihrem Wirklichkeitsgehalt ganz und gar bezweifelte Sprache und Sichtweise an- und einzunehmen, soll - beispielsweise - die soziale Kultur im antiken Griechenland zugänglich werden; eine Sichtweise, die eben auch uns Heutigen nicht vollkommen unvertraut ist. Was nicht zuletzt mit dem Hinweis auf die besondere, eigentümlich zwischen Imagination, freier Assoziation und aufmerksamer Sacherfassung oszillierende Erfahrung ästhetisch bedeutsamer Gegenstände zu belegen ist. Ästhetische Erfahrung ist nichts Archaisches, sondern genuin neuzeitlich, und trotzdem ist sie unterschieden und unterscheidbar vom Vollzug objektiver Wissenschaftserkenntnis, ein Faktum, das ihr dennoch nicht ihre (relative) Legitimität raubt. Dass ästhetische Erfahrungen und deren Anlässe vom Standpunkt wissenschaftlicher Rationalität aus in ihrer Verbindlichkeit radikal zu bezweifeln sind,

Zitiert nach: Kurt Hübner, Die Wahrheit des Mythos (München 1985), 67.

2 J.P. Vernant, Mythe et société en grèce ancienne, Paris 1974, 203. Die deutsche

Übersetzung zitiert nach Hübner, Die Wahrheit des Mythos, 79f.) 
besagt nichts; weder hinsichtlich ihres eigenen Wirklichkeitsgehaltes, noch hinsichtlich ihrer anthropologischen und sozialen Relevanz.

Wilamowitz-Möllendorf (nur deshalb habe ich die Anekdote erinnert) ist für beideVermögen, für die ästhetische Sensibilität und für die kulturerschliessende Hermeneutik, ein Exempel; nicht zufällig spricht er im zitierten Zusammenhang neben Herodot auch von den Gedichten Sapphos.

Die Kompetenz zu zweifeln ist ein konstitutives, unentbehrliches Instrument der Moderne. Das will ich nicht bestreiten. Wichtig ist lediglich, die begrenzte Reichweite moderner, d.h. wissenschaftlichkritischer Realitätsbestimmung zu konstatieren. Vergisst man sie, wird nicht nur die Vielfalt und anhaltende Koexistenz menschlicher Weltbezüge verkannt, am Ende ist man nicht einmal mehr in der Lage, die selber durchaus modernen Phänomene ästhetischer Theorie und Praxis zu begreifen.

Zweifel sind Fragen gesteigerter Intensität, besser: Fragen im Dringlichkeitsformat. Sie zielen mit bedingungsloser Energie auf sicheres Wissen. Das reine Offenhalten des Problems genügt ihnen nicht.

Auf einfache Fragen findet man Antworten. Zweifel beginnen dann, wenn die zur Verfügung stehenden Antworten unbefriedigend erscheinen; oder sich überhaupt keine Antworten einstellen. Zweifel gehören zu Wahlsituationen, die sich nicht entscheiden lassen: Was ist vorzuziehen, die Alternative A - oder nun doch B? weil unter den besonderen Umständen (die zuerst $\mathrm{zu}$ wenig in Betracht gezogen worden sind) A von Bedingungen abhängig ist, deren Eintreffen nun eher unsicher geworden ist ...? Und so weiter. Je nachdem, wie ausgeprägt die Angst vor dem Scheitern der möglichen Antwort ist, je nachdem, wie zuverlässig die Verhältnisse uns erscheinen, unter denen wir eine bestimmte Alternative als die richtige betrachten, verschwinden die Zweifel - oder nicht; und je nachdem bleibt unser intellektuelles Selbstvertrauen intakt - oder nicht.

Es ist offensichtlich: Zweifel und ihre Hartnäckigkeit sind - auch in hohem Mass abhängig von den subjektiven, personalen Eigenschaften der jeweils Zweifelnden.

Aber diesen Zusammenhang will ich, wie gesagt, hier nicht länger erörtern. Worum es mir jetzt geht, ist die Differenz von 
Zweifelsformen. Ich schlage vor, jedenfalls drei Arten auseinander zu halten: den einfachen Zweifel (wenn vorhandene Antworten zweifelhaft werden); das unglückliche Bewusstsein (wenn einer Person die Fähigkeit abhanden gekommen ist, ihren allemal unvollkommenen, aber plausibel dünkenden Annahmen zu trauen, jenen schwankenden »Gewissheiten", die für die menschliche Erkenntnissuche charakteristisch sind); den philosophischen Zweifel im eigentlichen Sinn.

"Philosophische Zweifel im eigentlichen Sinn« (so will ich sie an dieser Stelle und zum Zweck meiner »kleinen Notiz« definieren) betreffen die prinzipielle Möglichkeit endlicher Vernunftwesen, zwischen Doxa und wahrhafter Einsicht, zwischen blossem Glauben und wirklichem Wissen, zwischen subjektiver Überzeugung und objektiver Erkenntnis zu trennen. Man braucht keine grosse philosophiehistorische Informiertheit, um zu bemerken, dass die genannten Gegensätze der Philosophie des Abendlandes von Anfang an als Herausforderung dienten. Schon vor Platos Ideenlehre möchte Parmenides die Scheinhaftigkeit des Bewegten und der Bewegung klar machen, und auch Descartes'Absicht, durch den Radikalzweifel hindurch zum "fundamentum inconcussum«, zur beständigen Wahrheit und eindeutigen Wirklichkeit zu gelangen, verdankt sich der Konfrontation des Reichs der blossen Vermutungen und Glaubensannahmen mit dem Reich des realen Wissens.

"Fides quaerens intellectum« ist, nimmt man die abendländische Metaphysik nur von genügend weit weg in den Blick, nicht allein das Motto der christlichen Scholastik, sondern der Wahlspruch der westlichen Philosophie überhaupt. Das gilt bis zu Nietzsche (jedenfalls dann, wenn man die schon im Altertum heterodoxe Tradition des radikalen Skeptizismus ausblendet). Nietzsches Destruktion des Wahrheitsglaubens, die Entdeckung seines "tollen Menschen", dass es nichts ist mit dem Versprechen des platonischen Sokrates, aus dem Höhlenlabyrinth und seinen Schattenspielen aufzusteigen ins Offene des ursprünglichen Sonnenlichts - seit diesem Entsetzen darüber, dass "Gott tot» ist und "wir ihn getötet haben", seit dem Staunen darüber, "dass wir es konnten", ist die Trennung von Sein und Schein so relativ geworden, dass der philosophische Zweifel sich verwandelt hat: Indem er aus seiner metaphysischen Phase in die der pragmatistischen Epoche verschoben worden ist; in eine Epoche, die vielleicht auch die der (posttheologischen) Vernunftkehre genannt werden darf. 
DieVernunft und ihr rationaler Geist - gemessen an den Ansprüchen, wie sie der platonischen, cartesischen, hegelianischen Philosophie eigneten - sind bescheiden geworden. Das markiert ihre aktuelle Gestalt. Der radikale Zweifel der antiken Skepsis und erst recht der philosophische Zweifel der platonisch-christlichen Weltentwürfe haben sich in einen postmetaphysischen Fallibilismus zurückgezogen: Dass für als brauchbar geltende Theorien und Aussagen nicht mehr erwartet werden darf als "rationale Plausibilität«, ist allgemeiner Konsens. Rationale Plausibilität, also bis auf weiteres bestätigte, in einem Theorierahmen befestigteVermutung, genügt für die Arbeit der Wissenschaft und den Einsatz technologischer Konzepte. Und falls ein lange Zeit gut funktionierendes Paradigma der Welterklärung brüchig erscheint und unhandlich wird für die Weiterentwicklung von Forschungsbedürfnissen, dann muss man das Paradigma eben so rasch wie möglich ersetzen; durch einen neuen Ansatz, der besser zu den Bedürfnissen und unseren Praktiken und deren Erfahrungen passt. "Was ist daran problematisch?«, fragt der Pragmatist und findet: »nichts«. Für das, was ich oben den philosophischen Zweifel im eigentlichen Sinn genannt habe, bleibt so und im Grunde gar kein Raum mehr: Er ist sinnlos geworden.

Ist die Gegenwart also ein Nicht-Ort für diese Form des Zweifels und des Zweifelns geworden? Deshalb, weil in ihr das fundamentale Gegenmotiv, der metaphysische Ausgriff auf die wahrhafte, die unerschütterliche Erkenntnis - der Sinn der Suche »fides quaerens intellectum « - abhanden gekommen ist? vom »Tod Gottes« entwurzelt und darum selbst gestorben?

Ich zweifle nicht daran, dass die Lage so zu deuten ist. Doch das heisst nicht, dass philosophisches Denken nun arbeitslos geworden wäre. Denn an die Stelle des instrumentellen Zweifels tritt ein erneutes - "anfängliches" - Fragen. Ein sehr offenes und offenhaltendes, fast naives Nachdenken: Was ist das eigentlich - die menschliche Vernunft? Diese seltsame Begleiterin menschlicher Lebendigkeit, ohne die die menschliche Lebendigkeit nicht mehr menschlich wäre? Dieses Vermögen, das in reflexiver Betrachtung als unhintergehbar erscheint, als die Bedingung der Möglichkeit und als der erste Ursprung dafür, dass für uns - die "animales rationales" - überhaupt etwas als etwas da ist, d.h. zugänglich, begreif- und sprachlich bestimmbar wird?

Gewiss, das sind nicht die Perspektiven der Gehirnwissenschaften und der Neuropsychologie, sondern es ist die Hinsicht der transzendentalen Überlegung. Eine Form des Nachdenkens, die sich dem 
Objektivismus und Empirismus der Naturwissenschaft entzieht, aber deswegen nicht obsolet ist. Wer das Letztere behauptet, hat lediglich die als selbstverständlich vorausgesetzte Überzeugung verinnerlicht, dass der naturalistische Szientismus die einzig richtige, also die "wahrhaft wahre« Methode sei.

Das Fragen der Vernunft nach ihrem eigenen Anfang und das dadurch - vielleicht - veranlasste Staunen (»der« Vernunft über sie selber und derVernünftigen über ihr - im Grunde genommen - abgründig rätselhaftes Können) eröffnet dies, was ich in bewusst heideggerianischem Tonfall als "anfängliches« Fragen bezeichnet habe.

Was geschieht der Vernunft, wenn sie sich selber fragwürdig geworden ist? Offenbar eine Erfahrung der Dezentrierung: Die Einsicht in ihre, naturwissenschaftlich nicht greifbare Abkünftigkeit nötigt zur Revision ihres Selbstbewusstseins, zum Abschied von jeder SelbstHerrlichkeit; sei sie die der wissenschaftlichen Forschung oder die der in ihr selber kreisenden Reflexionsbewegung hegelianischen Musters. Der späte Heidegger hat - mit nicht selten hilflos anmutenden sprachlichen Verrenkungen - diesen Gedanken zu erkunden versucht und ihn unter dem Leitwort der "Kehre" in seine Geschichte der Metaphysik und ihrer »Verwindung« eingefügt.

Man mag das heideggersche Vokabular einleuchtend finden oder nicht. Dass mit ihm etwas von dem wahrnehmbar wird, was auf die andere Seite dessen verweist, was der pragmatistisch-fallibilistischen Ernüchterung zu Grunde liegt, darf man zugeben. Und sich umkehren: Um - statt dem alten, metaphysischen Zweifeln der Philosophie, das in der cartesianisch inspirierten Neuzeit seine Vollendung erfährt - dem (falls ein Prädikat nötig ist:) postmetaphysisch fragenden Staunen der Philosophie wieder Raum zu geben.

Ich sagte, der philosophische Zweifel sei das Primärwerkzeug der Moderne - und ihr »Kainszeichen«. Was damit gemeint ist, sollte klar geworden sein. Im Gebrauch des philosophischen Zweifels verbirgt sich entweder die Anmassung, die Vernunft absolut zu setzen und als Herrin der Welt und ihrer Geschichte zu etablieren, oder, komplementär dazu, eine total gewordene, also abgründige skeptische Verzweiflung. Hegels absoluter Geist und Nietzsches Zwillinge, der "tolle« und noch mehr der "Über-Mensch« sind allesamt Geschwister. Sprösslinge einer Selbstdeutung der menschlich-endlichen Vernunft, die zwar zu verstehen, doch nicht mehr zu erneuern ist. Dasselbe gilt 
freilich von der Reaktion der Antimodernen, deren Rückzug auf die dogmatische Sakralisierung von Tradition und Autoritätsmacht so einfach erklärbar wie letztlich unsinnig ist. (Das würde ich jetzt gern näher begründen, doch dann müsste ich fast noch einmal von vorn beginnen. Mit blossem Schulterzucken ist diese Position aber nicht zu erledigen.)

Was also statt dessen?

Was immer möglich war, und was sich aus guten Gründen in den vergangenen hundert Jahren bereits entwickelt hat: die Kombination von Pragmatismus und postmetaphysischer Nachdenklichkeit. Diese Verbindung zwischen zuversichtlicher, wissenschaftlich-praktischer Entängstigung der Welt mit einer über die eigenen Glaubensgewissheiten aufgeklärten Vernunft, die vor den Kontingenzen, die zu ihr gehören müssen, nicht die Augen verschliesst, sondern menschenfreundlich mit ihnen umzugehen weiss.

"Menschenfreundlichkeit" - bei diesem Wort wäre nun anzusetzen, sollte genauer darüber nachgedacht werden, weshalb es vernünftig sein kann, dass die Vernunft an die Vernunft glaubt. Durch die Beschäftigung mit dem Zweifel sind wir damit allerdings in ein neues Land (oder ein anderes Meer) geführt worden. Was jetzt der Grund dafür sein muss, die Sache abzuschliessen.

PS: Am Ende dieser kleinen Notiz zur grossen Erzählung vom philosophischen Zweifel und seiner Verwandlung ein Fazit oder "Summary«, von dem ich hoffe, dass es - zum Schluss gelesen - nicht so rätselhaft ist, wie es nach erster Lektüre erscheinen mag:

Von der Antike bis in die Moderne ist der Zweifel der Grenzwächter der scharfen Trennung zwischen Glauben und Wissen. In der Zeit der Nachmetaphysik hat ihn die - philosophische - Frage abgelöst. Was er unerbittlich auseinander halten wollte, das zeigt sie als Moment eines Ganzen, das ganz nie zu haben sein wird. 